\title{
Vorticity topology of vortex pair interactions at low Reynolds numbers
}

\author{
Andersen, Morten; Schreck, Cédric; Hansen, Jesper Schmidt; Brøns, Morten
}

Published in:

European Journal of Mechanics, B/Fluids

Link to article, DOI:

10.1016/j.euromechflu.2018.10.022

Publication date:

2019

Document Version

Peer reviewed version

Link back to DTU Orbit

Citation (APA):

Andersen, M., Schreck, C., Hansen, J. S., \& Brøns, M. (2019). Vorticity topology of vortex pair interactions at low Reynolds numbers. European Journal of Mechanics, B/Fluids, 74, 58-67.

https://doi.org/10.1016/j.euromechflu.2018.10.022

\section{General rights}

Copyright and moral rights for the publications made accessible in the public portal are retained by the authors and/or other copyright owners and it is a condition of accessing publications that users recognise and abide by the legal requirements associated with these rights.

- Users may download and print one copy of any publication from the public portal for the purpose of private study or research.

- You may not further distribute the material or use it for any profit-making activity or commercial gain

- You may freely distribute the URL identifying the publication in the public portal

If you believe that this document breaches copyright please contact us providing details, and we will remove access to the work immediately and investigate your claim. 


\title{
Vorticity topology of vortex pair interactions at low Reynolds numbers
}

\author{
Morten Andersen ${ }^{1 *}$, Cédric Schreck ${ }^{2}$, \\ Jesper Schmidt Hansen ${ }^{1} \&$ Morten Brøns ${ }^{3}$ \\ ${ }^{1}$ Department of Science and Environment, Roskilde University, DK. \\ ${ }^{2}$ Ecole Nationale Supérieure de Mécanique et Aérotechnique, FR \\ ${ }^{3}$ Department of Applied Mathematics and Computer Science, Technical University of Denmark, DK \\ *Corresponding author, moan@ruc.dk
}

August 16, 2018

\begin{abstract}
We investigate vortex merging at low Reynolds numbers from a topological point of view. We identify vortices as local extremal points of vorticity and follow the motion and bifurcation of these points as time progresses. We consider both two-dimensional simulations of the vorticity transport equation and an analytical study of the core growth model. The merging process of identical vortices is shown to occur through a pitchfork bifurcation and for asymmetric vortices one vortex merges with a saddle through a cusp (perturbed pitchfork) bifurcation. Excellent agreement between the core growth model and the numerical simulations is observed. For higher Reynolds numbers, filamentation becomes dominant hence limiting the predictive value of the core growth model. A complete investigation of merging in the core growth model is conducted for all possible vortex strengths. Simple, analytical expressions are derived for bifurcation curves, merging time, and vortex positions depending on systems parameters.
\end{abstract}

\section{Introduction}

For general viscous flows, it is surprisingly difficult to define a vortex in a rigorous way which is also simple to use, and no generally accepted definition exists. Here we just mention a few approaches. Jeong and Hussain [1995] discuss a number of vortex definitions based on the velocity gradient, the $\Delta$ criterion, the $Q$ criterion and the $\lambda_{2}$ criterion, which for twodimensional flow all are identical. More recently Haller [2005] has proposed a definition based on the strain acceleration tensor and another definition based on vorticity [Haller et al., 2016]. In these definitions a vortex is a region in the fluid fulfilling a certain inequality. An alternative approach is to consider the topology of the entire vorticity field and how it changes as the dynamics progresses. This immediately leads to focusing on the critical points where $\partial_{x} \omega=\partial_{y} \omega=0$. They organize the structure of the level curves of vorticity: Generically, the critical points are isolated and either extrema encircled by closed level curves or saddles with stable and unstable manifolds which divide the vortical regions from one another. Thinking of a vortex as a local extremum of vorticity is a natural generalization of a point vortex. Saddles may have no simple physical interpretation, but must be present for topological reasons. The 
(a)
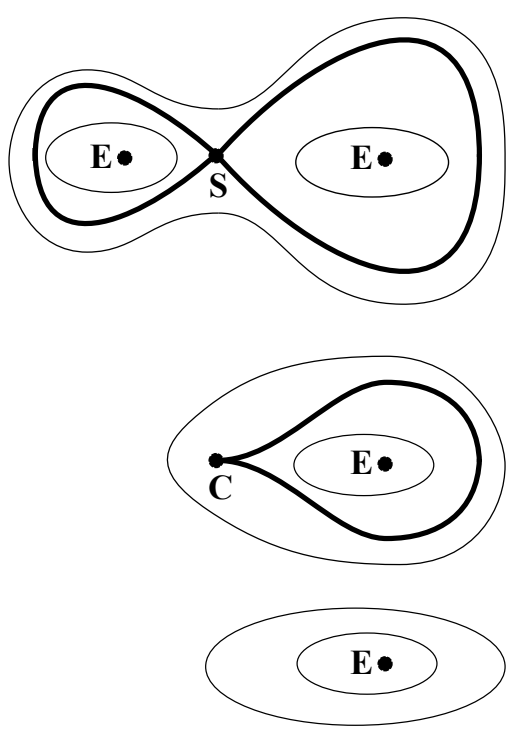

(b)

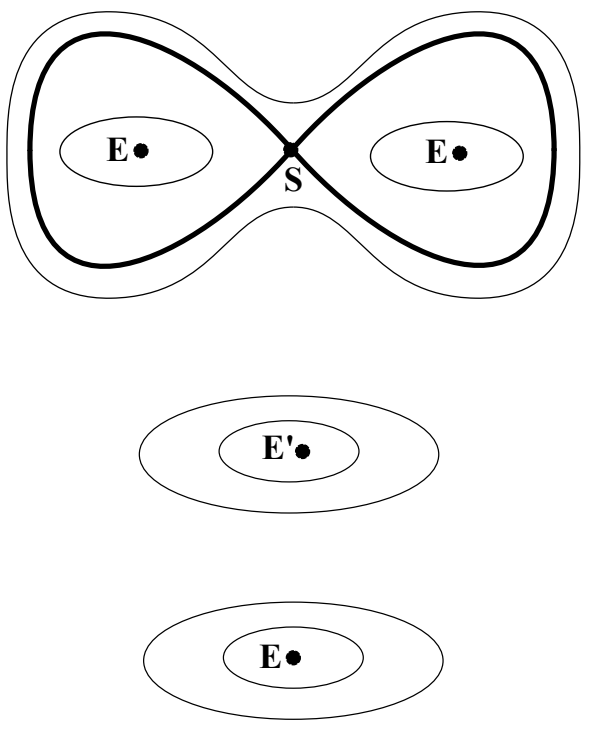

Figure 1: Bifurcation sequences in vortex merging. Typical level curves of vorticity are shown. Top row before bifurcation, middle row at bifurcation, bottom row after bifurcation. (a): Asymmetric merging, cusp bifurcation. The left extremum $E$ and the saddle $S$ merge to a cusp $C$ which disappears, leaving only the right extremum. (b): Symmetric merging, pitchfork bifurcation. The two extrema move toward the saddle. At the bifurcation, the three points merge into a degenerate extremum $E^{\prime}$, which after the bifurcation becomes a regular extremum.

interaction of critical points of vorticity has been used to describe the creation of the von Kármán vortex street [Heil et al., 2017].

A similar approach has been used extensively for the stream function rather than the vorticity. In that case the critical points are stagnation points and the level curves are simply the streamlines. See the reviews [Tobak and Peake, 1982, Brøns, 2007] and [Brøns et al., 2007, Balci et al., 2015] for specific applications to the cylinder wake and boundary layer eruption.

Understanding the interaction, creation and destruction of vortices is important for describing the dynamics of the flow and may be more instructive than the dynamics of the entire flow field. A classical example of this approach is the modeling of an inviscid flow by discrete point vortices, which reduces the problem to a set of ordinary differential equations for the vortex positions, see e.g. Newton [2001] or Meleshko and Aref [2007] for an introduction and a historical overview.

Interaction and merging of two vortices is a fundamental and well studied problem in fluid mechanics, see the recent review by Leweke et al. [2016] for an introduction and overview. Understanding vortex merging is needed for insight into complicated flows like wake structure of body-fluid interactions and two dimensional turbulence. Description of the vorticity topology of vortex merging is the purpose of the present paper. Bifurcation theory is used to study how critical points of vorticity interact and are created and destroyed, considering time as a bifurcation parameter - see figure 1 for typical merging sequences.

This approach is used to study vortex merging at low Reynolds numbers $(1 \leq \operatorname{Re} \leq 200)$. The method will be applied to simulation of the vorticity transport equation, and analytically 
to the core growth model which will be described below.

\subsection{Previous studies of vortex merging}

Early studies of vortex merging mainly focus on merging in inviscid fluids. Two point vortices in inviscid fluid cannot merge, as the vortex separation distance is constant. However, three point vortices may collapse in finite time (merge) depending on initial conditions and vortex strengths, [Synge, 1949, Aref, 1979]. Two or more vortex patches in inviscid fluid may lead to merging; Deem and Zabusky [1978] and Dritschel [1985] studied vortex patches with constant vorticity in inviscid fluid. This simplification implies that only the evolution of the vortex patch boundaries are needed to compute the evolution of the flow field, coined contour dynamics. Equilibrium solutions of such states are found, which is not possible in viscous fluid.

Some analytical investigations of merging in inviscid fluid restricting vortices to elliptical shapes and two symmetric vortices were conducted by Melander et al. [1988]. Merging of vortex patches with Gaussian profile in inviscid fluid is investigated and compared to laboratory experiments in rotating fluids by Trieling et al. [2005].

Cerretelli and Williamson [2003] describe vortex merging by four stages, where diffusive growth is the first, followed by a convective stage leading to the second diffusive stage and finally the merged diffusive stage. Brandt and Nomura [2007] also discuss four vortex merging stages in two - dimensional flow for symmetric vortex merging depending on the vorticity gradient and rate of strain. Brandt and Nomura [2010] investigate the situation with asymmetric vortices. A merging criterion for two-dimensional co-rotating vortices has been proposed by Meunier et al. [2002]. The criterion describes when a rapid decrease in the separation between the vortices (the onset of vortex merging) is observed and is given by a critical length scale related to the second moment of vorticity divided by the circulation. For the experimental part the early stage vortices are found to agree well with Gaussian vortices. Meunier et al. [2005] discuss two point vortices and two Gaussian vortices in relation to vortices behind an aircraft, which is also discussed by Jacquin et al. [2003]. Josserand and Rossi [2007] investigate vortex merging of co-rotating vortices at large Reynolds numbers. A recent contribution is the work by Folz and Nomura [2017] where numerical simulations are used to characterize interaction of two vortices with varying vortex strength ratios.

\subsection{The core growth model}

With one point vortex of strength $\Gamma$ as initial condition at $\boldsymbol{x}=\mathbf{0}$ (bold face denotes vector $\left.\boldsymbol{x}=(x, y) \in \mathbb{R}^{2}\right), \omega(\boldsymbol{x}, 0)=\Gamma \delta(\mathbf{0})$ the two-dimensional vorticity transport equation can be solved analytically yielding the Lamb - Oseen vortex [Saffman, 1992]

$$
\omega(r, t)=\frac{\Gamma}{\pi \sigma(t)^{2}} e^{-\frac{r^{2}}{\sigma(t)^{2}}}, \quad u_{\theta}=\frac{\Gamma}{2 \pi r}\left(1-e^{-\frac{r^{2}}{\sigma(t)^{2}}}\right), \quad u_{r}=0,
$$

with

$$
\sigma(t)=\sqrt{4 \nu t}
$$

This means that for $r \gg \sigma$ we have $u_{\theta} \approx \frac{\Gamma_{0}}{2 \pi r}$ which is the velocity generated by a point vortex of the same strength. The Lamb - Oseen vortex is an attracting solution for any integrable initial vorticity field as proved by Gallay and Wayne [2005]. Hence, unbounded flow with an integrable initial vorticity distribution with non zero total circulation will converge to 
a single Lamb - Oseen vortex after sufficiently long time. For multiple vortices as initial condition to the vorticity transport equation, an explicit analytical solution is typically not available. Therefore, we resolve to numerical simulations of the vorticity transport equation and compare these to analytical investigations of a synthetic flow given by the core growth model, also known as the multi Gaussian model. Here, the centers of the Gaussian vortices move in a field prescribed by the other vortices (similar to inviscid point vortex dynamics) and the cores of the vortices diffuse as predicted by the Lamb - Oseen solution of a single vortex. Investigations of the core growth model with few vortices are conducted by Jing et al. [2010] but the idea of splitting advective and diffusive mechanisms in computations with Gaussian vortices is an older approach [Beale and Majda, 1981, Kida and Nakajima, 1998].

In the case of $N$ Gaussian vortices with strengths $\Gamma_{k}$, the velocity of the $j$ th vortex center is (with complex notation $z=x+i y$, and ${ }^{*}$ indicating complex conjugate)

$$
\dot{z}_{j}^{*}=\frac{1}{2 \pi i} \sum_{k \neq j} \frac{\Gamma_{k}}{z_{j}-z_{k}}\left(1-\exp \left(-\frac{\left|z_{j}-z_{k}\right|^{2}}{4 \nu t}\right)\right),
$$

and the velocity field at any point, $z$, in the fluid that is not a center of a Gaussian vortex is

$$
\dot{z}^{*}=\frac{1}{2 \pi i} \sum_{k=1}^{N} \frac{\Gamma_{k}}{z-z_{k}}\left(1-\exp \left(-\frac{\left|z-z_{k}\right|^{2}}{4 \nu t}\right)\right) .
$$

The vorticity field can be computed when the location of the Gaussian vortex centers, $z_{k}(t)$, is known by the formula

$$
\omega=\sum_{k=1}^{N} \frac{\Gamma_{k}}{4 \pi \nu t} \exp \left(-\frac{\left|z-z_{k}\right|^{2}}{4 \nu t}\right) .
$$

Thus, in the core growth model the vorticity is calculated from a superposition of Gaussian terms. In general, this is not an exact solution of the vorticity transport equation but we will regard it as a simple model allowing some viscous effects. Gallay [2011] proved (under certain conditions) that the solution to the vorticity transport equation converge to a sum of Lamb Oseen vortices for viscosity going to zero and for finite time when the inital condition is a sum of point vortices. A related result was obtained by Marchioro [1998]. The first diffusive stage of vortex merging described by Cerretelli and Williamson [2003] is found to be well described by two Lamb - Oseen vortices, hence there is experimental justification for investigating the core growth model in detail. The core growth model with a few vortices can be analyzed in detail and may show qualitative information that is valuable also for more realistic flow.

Importantly, the single, Lamb - Oseen vortex, Eq. (1), has the property that $\boldsymbol{u} \cdot \nabla \omega=0$ hence it is also a solution to the diffusion equation

$$
\partial_{t} \omega=\nu \nabla^{2} \omega
$$

As this is a linear equation, a sum of Lamb - Oseen vortices is a solution to this equation. Therefore, the core growth model is expected to perform well when the advection term $\boldsymbol{u} \cdot \nabla \omega$ is not dominating.

\subsubsection{Previous studies of the core growth model}

Core growth models with few vortices have been subject to little attention and analysis despite a Gaussian vortex profile being observed in experiments [Trieling et al., 2010]. In Jing 
et al. [2010] the instantaneous streamlines and vorticity contours of three, collinear Gaussian vortices are investigated. The three circulations are chosen such that the three vortices stay aligned and the whole configuration rotates with time dependent rotation rate. Numerical analysis of the core growth model is compared to numerical solution of the vorticity transport equation and both indicate a linear increase of merging time with Reynolds number.

Kim and Sohn [2012] derive the integrals of motion for the core growth model similar to point vortex dynamics and a time-dependent Hamiltonian for the position of the vortex centers is derived. They prove the impossibility of self similar collapse of three vortices in the core growth model. This is a result contrary to the point vortex case where self similar collapse is possible [Aref, 1979].

Jing et al. [2012] investigate vortex interaction using the core growth model of two vortices, one case of equal vortex strength and one case of asymmetric vortex strength. Their main focus is on analyzing a suitable rotating coordinate system, where time dependent separatrices of the instantaneous streamlines can be computed with a rich dynamics that is related to the passive tracer evolution of the system. Here, we will complement their investigations by studying all possible two vortex interactions in the core growth model and compare the results to simulations of the vorticity transport equation. To our knowledge this is the first analytical investigation of the vorticity topology of the core growth model. Recall, an extremum of the vorticity is considered a vortex. The description of a vortex as a single point allows bifurcation analysis to describe the merging of the vortices. In the core growth model, all bifurcations are investigated and described and simple equations for the position of the vortex extrema as a function of time and system parameters are derived.

The paper is organised as follows: In Section 2 the numerical simulation of the vorticity transport equation is described. In Section 3 the topologies and bifurcation curves of the core growth model are investigated after a useful scaling and coordinate system are chosen. The analytical results are compared to the numerical results of the simulation of the vorticity transport equation. In Section 4 equations for trajectories of the vortices are derived and compared to numerical results. In Section 5, the results are summarized and compared to previous results in the literature, and finally alternative approaches are discussed.

\section{Methodology}

\subsection{Scaling and parameters}

We consider two Gaussian vortices. Vortex 1 with strength $\Gamma_{1}$ is situated at $(-d, 0)$ and vortex 2 with strength $\Gamma_{2}$ is situated at $(d, 0)$. We let $\Gamma_{2}>0$ which does not exclude any case - we can still investigate opposite signed circulations of the two vortices as well as circulations with the same sign. Two vortices with negative circulation rotate in opposite direction to two vortices with positive sign. Likewise, a sign change in $\Gamma_{1}+\Gamma_{2}$ implies a sign change in the direction of rotation. To obtain the vorticity transport equation in non dimensional form, length and time scales are divided by typical quantities. Following the approach of Meunier et al. [2002] and Jing et al. [2012] the typical quantities are obtained from the point vortex formulation of the problem. The typical length scale, $L$, is the distance between the vortices and the typical time, $T$, is the time of one revolution of the two point vortices

$$
T=\frac{(2 \pi)^{2}(2 d)^{2}}{\left|\Gamma_{1}\right|+\Gamma_{2}} .
$$


The non dimensional time in the vorticity transport simulations is then $\bar{t}=t T^{-1}$. The advective forces scale with $\Gamma$ and the viscous forces scale with $\nu$. The Reynolds number of the simulations is defined as

$$
\operatorname{Re}=\frac{\left|\Gamma_{1}\right|+\Gamma_{2}}{2 \nu}
$$

which is consistent with the Reynolds number used in Meunier et al. [2002] and Jing et al. $[2012]$ being $\frac{\Gamma}{\nu}$ for two equal vortices.

\subsection{Numerical method}

We integrate the vorticity transport equation in (arbitrary) dimensional form, as this allows for a direct comparison with the model, using the variable transformations given above. As the choice of units are arbitrary, we will not write these explicitly. The Reynolds number is varied by varying the kinematic viscosity $\nu$ since $\Gamma_{1}+\Gamma_{2}=2$ in all simulations. As Re $\leq 200$ and we have a square domain, a simple finite difference method with the explicit Euler integrator scheme suffices. Let $\mathbf{u}=(u, v)$ be the velocity field, then for any grid point $i, j$ in the system domain we have

$$
\begin{aligned}
\omega_{i j}^{n+1}(\Delta t) & =\omega_{i j}^{n}-\left(\mathbf{u}_{i j}^{n} \cdot\left(\nabla \omega_{i j}^{n}\right)-\nu \nabla^{2} \omega_{i j}^{n}\right) \Delta t \\
\nabla^{2} \psi_{i j}^{n+1} & =-\omega_{i j}^{n+1}, \quad u_{i j}^{n+1}=\frac{\partial \psi_{i j}^{n+1}}{\partial y}, \quad v_{i j}^{n+1}=-\frac{\partial \psi_{i j}^{n+1}}{\partial x},
\end{aligned}
$$

where $n$ is the time iteration index and $\Delta t$ is the integrator time step. The spatial derivatives are approximated by central differences. The Euler scheme is only conditionally stable and the appropriate time step size is dependent on the cell Reynolds number [E and Liu, 1996b]. Therefore, we apply an adaptive time step method, where the error estimator is given by the maximum of the absolute differences, i.e. $\operatorname{err}=\max \left\{\left|\omega_{i j}^{n}(\Delta t)-\omega_{i j}^{n}(\Delta t / 2)\right|\right\}$; the relative tolerance is set to $\leq 0.1 \%$. The computational domain is $[-6 ; 6] \times[-6 ; 6]$ using grid spacing $\Delta x=0.06$, and we have applied periodic boundary conditions. The numerical solver is readily implemented in GNU Octave [Eaton et al., 2016]. In particular, the Poisson equation is solved also using a finite difference scheme and GNU Octave's build-in direct matrix solver (Hansen [2011], Eaton et al. [2016]). The Gaussian vortices are placed at $( \pm 1,0)$ and the initial condition is

$$
\omega(\boldsymbol{x}, 0)=\Gamma_{1} \exp \left(-\frac{(x+1)^{2}+y^{2}}{\sigma_{0}^{2}}\right)+\Gamma_{2} \exp \left(-\frac{(x-1)^{2}+y^{2}}{\sigma_{0}^{2}}\right),
$$

with $\sigma_{0}=\Delta x$ unless otherwise stated.

The numerical method was tested in different ways. (i) For $R e=100$ results from the first order Euler scheme was compared against the 4th order Runge-Kutta algorithm [E and Liu, 1996b,a]. With the same error tolerance the average time step is approximately $10 \%$ larger for the 4th order Runge-Kutta scheme, however, this does not compensate for the factor of four in computational cost. (ii) To test for finite size effects, the domain was increased by a factor 2 for $R e=100$ giving the same merging time. (iii) The effect of grid point spacing was tested by comparing the vorticity field for spacings $\Delta x=0.12,0.09$ and 0.06 . The difference is expected to be largest in the beginning of the simulation as the gradients are largest for small times. 
After 200 iterations the difference in vorticity extrema for $\Delta x=0.12$ and $\Delta x=0.06$ is $1 \%$, no difference for $\Delta x=0.09$ and 0.012 is found to five decimal places, that is, the relative error is less than $10^{-3 \%}$. The same conclusion is drawn for the position of the vorticity extrema. The difference decreases as time increases as expected. The exact differences are dependent on initial conditions, and the initial vorticity must be a distribution with a finite width. As noted above we use the smallest grid spacing, $\Delta x=0.06$, in order to achieve high resolution and thereby minimizing the numerical error when interpolating data for the location of the vorticity extrema.

\section{$3 \quad$ Vorticity topology in the core growth model}

Let the center of vortex 1 at $\left(x_{1}(t), y_{1}(t)\right)$ be advected in the velocity field from vortex 2 at $\left(x_{2}(t), y_{2}(t)\right)$ prescribed by Eq. (1) and vice versa, and let their initial locations be at $(-d, 0)$ and $(d, 0)$. One can deduce [Kim and Sohn, 2012] that the distance between the vortices is conserved, similar to the point vortex case. The center of vorticity, $z_{c v}$, is stationary (using $z=x+i y$ ) being

$$
z_{c v}=\frac{\alpha z_{1}+z_{2}}{1+\alpha}, \quad \alpha \neq-1
$$

where the parameter $\alpha$ is

$$
\alpha=\frac{\Gamma_{1}}{\Gamma_{2}} .
$$

The center of vorticity moves infinitely far away for $\alpha$ approaching -1 . Both Gaussian vortices rotate around $z_{c v}$ with identical time-dependent angular velocites,

$$
\frac{\mathrm{d} \phi}{\mathrm{d} t}=\frac{\Gamma_{1}+\Gamma_{2}}{2 \pi(2 d)^{2}}\left(1-\exp \left(-\frac{(2 d)^{2}}{\sigma(t)^{2}}\right)\right),
$$

where $\sigma(t)$ is given by Eq. (2). For small times the well known expression for the angular

velocity of two point vortices is obtained while for large time the viscosity makes $\frac{\mathrm{d} \phi}{\mathrm{d} t}$ go to zero. The time dependent vorticity is given by

$$
\begin{gathered}
\omega=\frac{\Gamma_{1}}{\pi \sigma(t)^{2}} \exp \left(-\frac{\left(x-x_{1}(t)\right)^{2}+\left(x-y_{1}(t)\right)^{2}}{\sigma(t)^{2}}\right)+ \\
\frac{\Gamma_{2}}{\pi \sigma(t)^{2}} \exp \left(-\frac{\left(x-x_{2}(t)\right)^{2}+\left(y-y_{2}(t)\right)^{2}}{\sigma(t)^{2}}\right) .
\end{gathered}
$$

Since the Gaussian vortices move on concentric circles with the same angular velocity, one can choose new coordinates $\left(x^{\prime}, y^{\prime}\right)$ by a translation and a rotation such that $\left(x-x_{1}(t)\right)^{2}+$ $\left(y-y_{1}(t)\right)^{2}$ is mapped to $\left(x^{\prime}+d\right)^{2}+y^{\prime 2}$ and $\left(x-x_{2}(t)\right)^{2}+\left(y-y_{2}(t)\right)^{2}$ is mapped to $\left(x^{\prime}-d\right)^{2}+$ $y^{\prime 2}$. This guarantees that the vorticity topology can be studied with the centers of the two Gaussian vortices at fixed positions, which facilitates the analysis.

Dimensionless variables (denoted ${ }^{\sim}$ ) are introduced by $x^{\prime}=d \tilde{x}, y^{\prime}=d \tilde{y}, \omega=\frac{\Gamma_{2}}{\pi \sigma^{2}} \tilde{\omega}$ and the dimensionless time, $\tau$

$$
\tau=\frac{4 \nu}{d^{2}} t .
$$


The relation between the dimensionless time, $\bar{t}$, used in the vorticity transport simulations and $\tau$ is

$$
\bar{t}=\frac{\operatorname{Re}}{32 \pi^{2}} \tau .
$$

The time $\bar{t}$ is useful for comparing to existing, numerical results while the time, $\tau$, simplifies the analysis of the core growth model.

We now relabel $(\tilde{x}, \tilde{y})$ to $(x, y)$ i.e. the dimensionless coordinates in the corotating coordinate system while $\left(x_{l a b}, y_{l a b}\right)$ refer to the dimensionless coordinates in the lab frame, where the vorticity transport simulations are performed. The dimensionless vorticity in the core growth model becomes

$$
\omega=\alpha \exp \left(-\tau^{-1}\left((x+1)^{2}+y^{2}\right)\right)+\exp \left(-\tau^{-1}\left((x-1)^{2}+y^{2}\right)\right) .
$$

Symmetry implies

$$
\omega(x, y, \alpha, \tau)=\omega(x,-y, \alpha, \tau)
$$

and

$$
\omega(x, y, \alpha, \tau)=\alpha \omega\left(-x, y, \frac{1}{\alpha}, \tau\right) .
$$

Both the vorticity transport simulations and the core growth model have the initial Gaussian vortices placed at $( \pm 1,0)$. For the vorticity transport simulations the advective time scale related to point vortex motion is used and for the core growth model a viscous time scale is used.

The topology of the vorticity given by Eq. (17) will be investigated for any non zero $\alpha$, and positive, increasing $\tau$. By Eq. (19) it is sufficient to investigate $|\alpha| \geq 1$. To proceed, the critical points of Eq. (17) are computed, satisfying $\partial_{x} \omega=\partial_{y} \omega=0$, the type (saddle or extremum) is determined by the sign of the Hessian determinant $|H|=\partial_{x x} \omega \cdot \partial_{y y} \omega-\left(\partial_{x y} \omega\right)^{2}$ at such a point. See Fig. 2 for the typical cases. The condition $|H|=0$ at a critical point indicates a bifurcation point. The location of non degenerate critical points $(|H| \neq 0)$ is described in the following theorem - see Appendix A for a proof.

Theorem 1. Location of critical points

- All critical points occur on the $x$-axis.

- For vortices of same sign, $\alpha>0$, critical points only occur for $|x|<1$ and for vortices of opposite sign, $\alpha<0$, critical points only occur for $|x|>1$.

- In the generic case $\partial_{x x} \omega \neq 0$ at a critical point, the type of the critical point is known. The critical point closest to $x= \pm 1$ is an extremum, and the consecutive points are of alternating type.

\subsection{Bifurcation analysis}

The expression for $\partial_{x} \omega(x, 0)$ may be rearranged to

$$
\frac{\tau \partial_{x} \omega(x, 0)}{2(x+1) \exp \left(-\tau^{-1}\left((x+1)^{2}+y^{2}\right)\right)}+\alpha=g(x, \tau)
$$


(a)

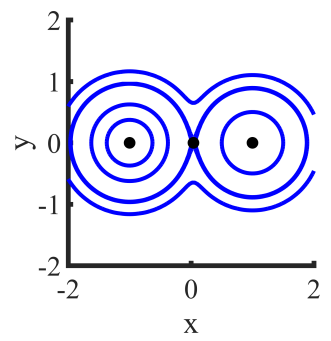

(b)

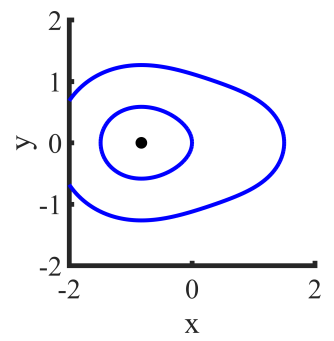

(c)

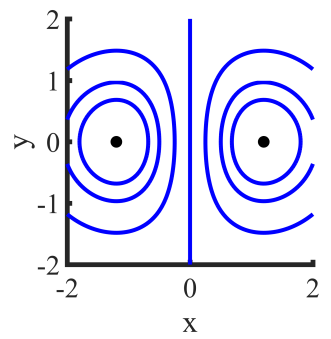

Figure 2: Examples of the three structurally stable vorticity topologies in the core growth model. (a) $\alpha=2, \tau=0.2$, (b) $\alpha=2, \tau=2$, (c) $\alpha=-1, \tau=2$.

with

$$
g(x, \tau)=-\frac{x-1}{x+1} \exp \left(4 \tau^{-1} x\right) .
$$

Then $\partial_{x} \omega(x, 0)=0$ corresponds to $\alpha=g(x, \tau)$. The motivation for introducing $g$ is a decoupling of the parameters, such that critical points are solutions of $\alpha=g(x, \tau)$ and bifurcation points, which must fulfill $\partial_{x} \omega=\partial_{x x} \omega=0$, are characterized by $\partial_{x} g(x, \tau)=0$. The graphical interpretation of a bifurcation point is a horizontal tangent of $g$. The physical interpretation of a bifurcation for increasing $\tau$ is a merging of a saddle and at least one extremum of the vorticity, hence the bifurcation value is the merging time, $\tau_{m}$. See Fig. 2 and 3 for the interpretation of $g$ in some typical cases.

Three graphs of $g$ can be seen in Fig. 3. By computing the local minimum and the local maximum of $g$ for varying $\tau$ Fig. $4 \mathrm{a}$ is constructed. This can be achieved by an explicit formula, since zeros of $\partial_{x} g(x, \tau)$ corresponds to zeros of $p(x)$ given by

$$
p(x)=x^{2}-1+\frac{1}{2} \tau,
$$

which has two zeros for $\tau<2$

$$
x_{ \pm}(\tau)= \pm \sqrt{1-\frac{1}{2} \tau}
$$

showing that any bifurcation point occurs for $|x|<1$ i.e. for positive $\alpha$. The merging time, $\tau_{m}$, for $\alpha>1$ is then given by inserting $x_{+}$in Eq. (21) with $\tau=\tau_{m}$

$$
\alpha\left(\tau_{m}\right)=-\frac{x_{+}-1}{x_{+}+1} \exp \left(4 \tau_{m}^{-1} x_{+}\right)
$$

which inversely defines the merging time, and is illustrated in Fig. 4(a). The right hand side of Eq. (24) is a decreasing function of $\tau_{m}$, which goes to infinity for $\tau_{m}$ going to 0 and goes to 1 for $\tau_{m}$ going to 2 . Hence, three critical points are most persistent for $\alpha=1$. Here, the bifurcation occurs at $\tau_{m}=2$. The symmetry in Eq. (19) provides a similar argument for $x_{-}$ and $0<\alpha<1$. Exactly when $\alpha=1$ the observed bifurcation is a pitchfork bifurcation. For all other values of $\alpha$ the bifurcation correspond to the perturbed pitchfork bifurcation - see Fig. 6. The findings are summarised in the following theorem (proved in Appendix A).

Theorem 2. Bifurcations of vorticity contours.

- There are 1,2 or 3 critical points of vorticity for $\alpha>0$. 


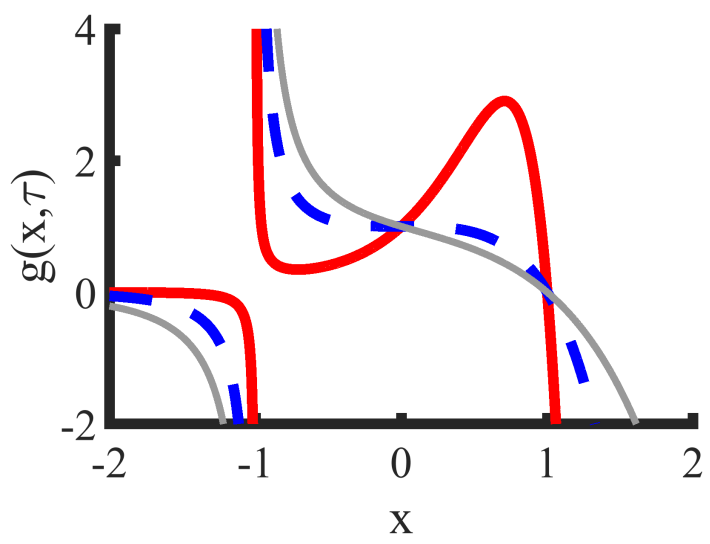

Figure 3: Plots of $y=g(x, \tau)$ for $\tau=1$ (red), $\tau=2$ (dashed blue), $\tau=3$ (grey). Critical points of vorticity are intersections of the horizontal line $y=\alpha$ for fixed $\alpha$ and $y=g(x, \tau)$. Consider first the red curve. For $\alpha$-values between the two local extrema of $g$ there are three critical points corresponding to Fig. 2(a). At $\tau=2$ the two local extrema merge, hence for any $\alpha>0$ there is only one solution to $\alpha=g(x, \tau)$ giving the final topology in Fig. 2(b). For any $\tau>0$ and $\alpha<0$ topology Fig. 2(c) appears with two extrema and no saddles.

(a)

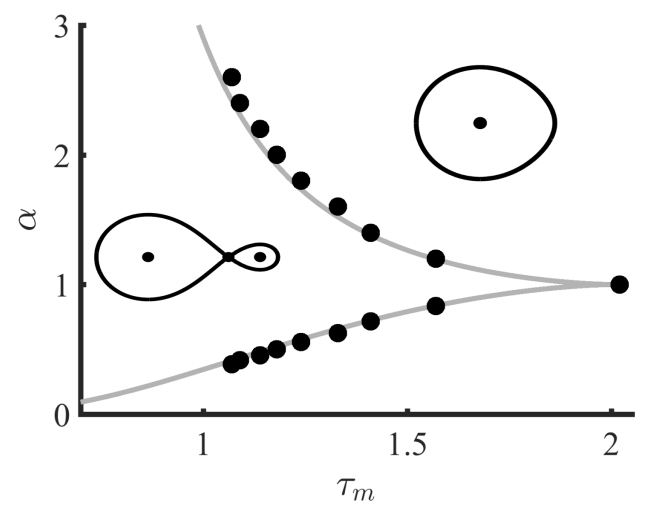

(b)

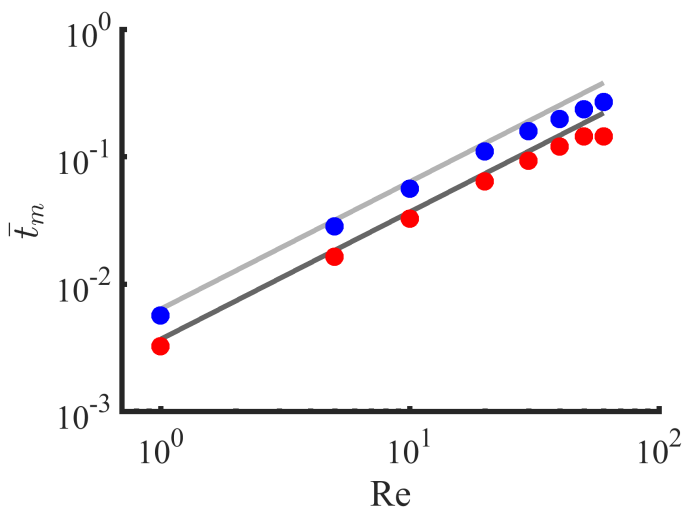

Figure 4: The analytical relation between merging time and $\alpha$, Eq. (24), is the grey curve in (a) and black dots are simulation results $(\mathrm{Re}=5)$. In $(\mathrm{b})$ blue dots are simulations of the vorticity transport equation with $\alpha=1$, red dots correspond to $\alpha=2$. The upper full line is a plot of Eq. (26) with $\alpha=1$ and the lower full line is a plot of Eq. (26) with $\alpha=2$. 
- For increasing time, three critical points merge to one critical point for $\alpha>0$. This may happen through a pitchfork bifurcation $(\alpha=1)$ or a perturbed pitchfork bifurcation $(\alpha \neq 1)$.

- The final topology is one vortex extremum for $\alpha>0$. An upper limit of the merging time is

$$
\tau_{\max }=2
$$

This upper limit is realized in the case $\alpha=1$.

- There are no bifurcations for vortices of opposite sign. Two extrema are the only critical points of the vorticity.

In Fig. 4 the model prediction of merging time is compared to vorticity transport simulations showing good agreement for low Reynolds numbers. The merging time in the simulations is predicted to be linearly increasing by Eq. (16)

$$
\bar{t}_{m}=\frac{\operatorname{Re}}{32 \pi^{2}} \tau_{m} .
$$

The simulations support the symmetric case $\alpha=1$ to be the most robust case of two vortices. For high Reynolds numbers, the core growth model overestimates the merging time. This is related to the filamentation of the vortices appearing in the vorticity transport equation which is not part of the core growth model (see the discussion, Section 5).

\section{Trajectories of critical points in the core growth model}

In dimensionless variables the center of vorticity given by Eq. (11) is

$$
x_{c v}=\frac{1-\alpha}{1+\alpha} .
$$

In the laboratory frame, the critical points of vorticity rotate around $\left(x_{c v}, 0\right)$ with angular velocity given by Eq. (13). In dimensionless time, this becomes

$$
\frac{d \phi}{d \tau}=\frac{\operatorname{Re}}{16 \pi}\left(1-\exp \left(-4 \tau^{-1}\right)\right)
$$

While the merging time only depends on $\alpha$ and the initial core width corresponding to $\tau_{0}$, the rotation rate has a prefactor involving an extra parameter, Re. Hence, in the core growth model, merging time and topology is independent of Re. We will now consider trajectories, where Re does matter. Since merging occurs for $\tau \leq 2$ one can with less than $4 \%$ error in $\phi$ skip the exponential term in Eq. (28) to get

$$
\phi_{\text {approx }}=\frac{\operatorname{Re}}{16 \pi} \tau .
$$

Hence, the viscous slowdown is negligible, rendering the point vortex angle a good approximation, $\phi_{\text {approx }}=2 \pi \bar{t}$. For any fixed $\alpha, \tau_{m}$ is uniquely determined by Eq. (24) meaning that varying Re gives a linear increase in $\phi$ by Eq. (29). For any fixed $\alpha, \tau=\tau_{m}$ is fixed hence 
(a)

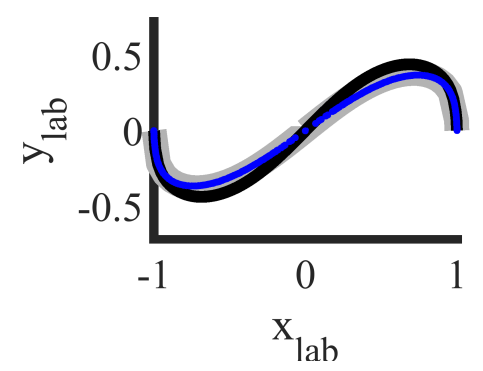

(c)

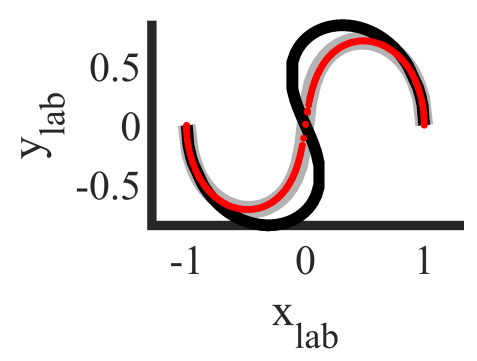

(b)

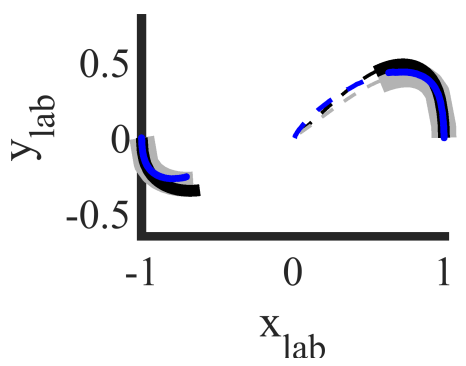

(d)

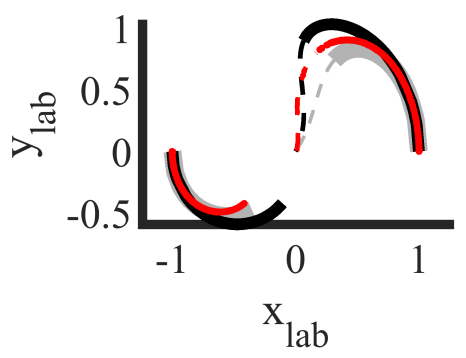

Figure 5: Trajectories of critical points of vorticity in the lab frame. The first column is the symmetric case, $\alpha=1$, and the last column illustrates an asymmetric case $\alpha=2$. Full curves correspond to vorticity extrema and the dashed curves are vorticity saddles. Blue curves are vorticity transport simulations at $\operatorname{Re}_{v t}=20$, red curves are $\operatorname{Re}_{v t}=50$. The black curve is the core growth model with $\operatorname{Re}_{\text {model }}=\mathrm{Re}_{v t}$. The grey curve in the top row is the core growth model with $\mathrm{Re}_{\text {model }}=17$, in the bottom row $\mathrm{Re}_{\text {model }}=37$. From the merging angle in the numerical simulation, $\mathrm{Re}_{\text {model }}$ is easily computed by Eq. (30) which is used to generate the grey curves for $\alpha=1$. 
(a)

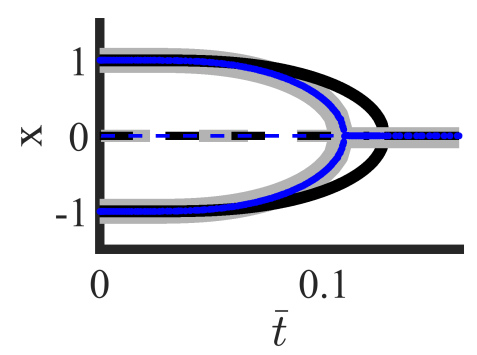

(c)

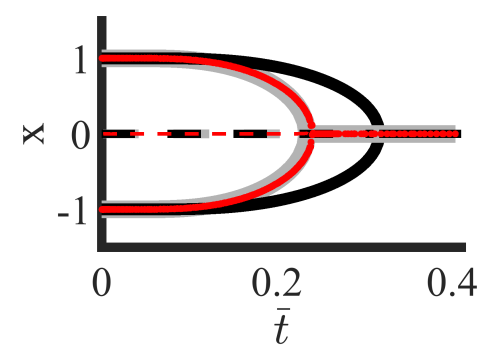

(b)

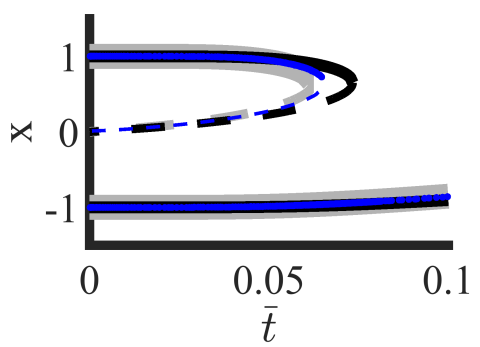

(d)

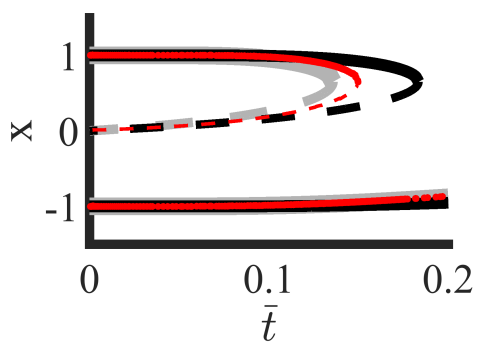

Figure 6: Position of the critical points of vorticity in the co-rotating frame (where all critical points are located on the $x$ - axis) as a function of time. Legends are similar to Fig. 5. Left column shows a pitchfork bifurcation, right column shows the perturbed pitchfork (cusp) bifurcation.

the angle at merging, $\phi_{m}$, scales linearly with Re. Recall, that for symmetric vortices, $\alpha=1$, the merging time is 2 hence a relation between Re and merging angle is by Eq. (29)

$$
\operatorname{Re}=8 \pi \phi_{m}, \quad \text { for } \alpha=1
$$

Re can thereby be estimated by observing the merging angle in an experiment. This approach is used in Fig. 5 for the grey curves.

The equation $\alpha=g(x(\tau), \tau)$ implicitly defines $x=x(\tau)$ when knowing an initial condition $\left(\tau_{0}, x\left(\tau_{0}\right)\right)$. This implies $\frac{d x}{d \tau}$ can be computed by implicit differentiation of $\alpha=g(x(\tau), \tau)$ giving

$$
\frac{d x}{d \tau}=\frac{x\left(x^{2}-1\right)}{\tau\left(x^{2}-1+\frac{1}{2} \tau\right)},
$$

which is valid as long as $\partial_{x} g(x, \tau) \neq 0$ hence when $p(x) \neq 0$ i.e. when no bifurcation occurs. The motion of the critical points in the co-rotating frame when no bifurcations occur is then dictated by Eq. (31) with $y=0$ and the initial condition $\left(\tau_{0}, x\left(\tau_{0}\right)\right)$. The trajectory of each critical point can thereby be found by solving a single ordinary differential equation.

In the laboratory frame, $\left(x_{l a b}(\tau), y_{l a b}(\tau)\right)$ is given by $x(\tau)$ from Eq. (31) combined with a rotation around $z_{c v}$ 


$$
\begin{aligned}
& x_{l a b}(\tau)=x(\tau) \cos \phi+\frac{1-\alpha}{1+\alpha}(1-\cos \phi) \\
& y_{l a b}(\tau)=x(\tau) \sin \phi-\frac{1-\alpha}{1+\alpha} \sin \phi,
\end{aligned}
$$

where $\phi=\phi_{\text {approx }}$ can be used for $\tau \leq 2$ i.e. to study merging. For the long term behavior of opposite signed vortices, the time evolution in Eq. (28) must be included. Simulations of the vorticity transport equation can be visualized in the co-rotating frame by using the inverse of Eq. (32)

$$
x=x_{l a b} \cos \phi+y_{l a b} \sin \phi+\frac{1-\alpha}{1+\alpha}(1-\cos \phi),
$$

which is used in Fig. 6.

The fixed point values of Eq. (31) are $x=-1,0,1$ which means that $[-1,0]$ and $[0,1]$ are trapping regions. A critical point cannot escape such a region if it is once in there.

A stationary critical point requires $\frac{d x}{d \tau}=0$ such that, at some time, $x=0$ or $x=1$ or $x=-1$. The two latter cases are not possible for finite, non zero $\alpha$. The case $x=0$ can only be obtained for $\alpha=1$ being the symmetric pitchfork - see Fig. 6 . The following theorem is a direct consequence of Eq. (31), see Appendix A for a proof.

Theorem 3. Dynamics of critical points for $\alpha>0$.

Survival of the strongest: If $\alpha \neq 1$ the weaker vortex merges with the saddle point for increasing time.

As an alternative to Eq. (31) $\alpha=g(x, \tau)$ can be reformulated such that $\tau$ is given in terms of $x$ i.e. as the inverse function, which can be used to compute $(\tau, x(\tau))$

$$
\begin{aligned}
& \tau=\frac{4 x}{\ln \alpha+\ln \left(\frac{1+x}{1-x}\right)}, \quad \text { for } \alpha>0, \text { i.e. }|x|<1, \quad(\alpha, x) \neq(1,0) \\
& \tau=\frac{4 x}{\ln (-\alpha)+\ln \left(\frac{x+1}{x-1}\right)}, \quad \text { for } \alpha<0, \text { i.e. }|x|>1 .
\end{aligned}
$$

For a given Re and $\alpha$, a range of $x$ - values may be inserted in Eq. (34) to get $(\tau, x(\tau))$, then Eq. (32) can be applied to get the location of the critical points of vorticity in the lab frame, see Fig. 5. This is a very simple procedure compared to conducting direct numerical simulation of the vorticity transport equation or actual experiment followed by post processing to find the critical points of vorticity.

\subsection{Dynamics of critical points of vorticity for $\alpha<0$}

The main focus of the present paper is the study of vortex merging. For opposite signed vortices $(\alpha<0)$ there are no bifurcations in the core growth model which limits the interest of this case, as the topology shown in Fig. 2(c) persists. Trajectories of the vorticity extrema may still be found. There is need for considering times longer than $\tau=2$ contrary to the case $\alpha>0$. Simple expressions and asymptotic forms of the trajectories of the vorticity extrema are stated in this section. The following theorem follows from Eq. (31) and Eq. (34), proved in Appendix A. 
(a)

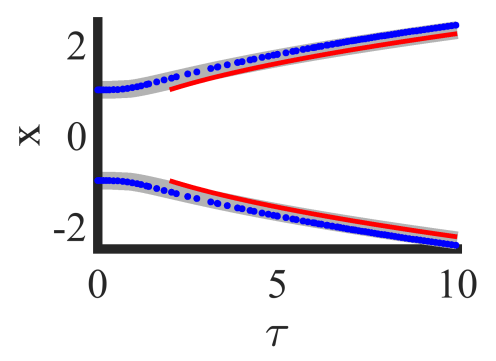

(b)

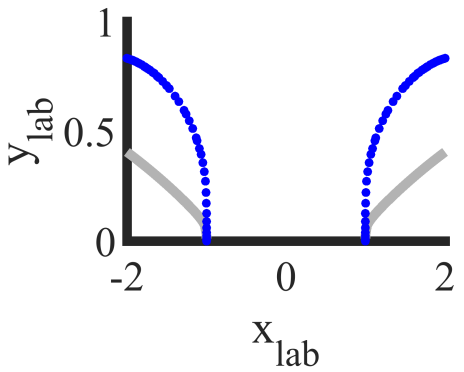

Figure 7: For $\alpha<0$, the core growth model has two persistent vorticity extrema and no saddles, which is also the case observed in vorticity transport simulations (blue dots). The location of the critical points of vorticity is seen in (a) for $\operatorname{Re}=20, \alpha=-1$. The red curve is the asymptotic formula $x= \pm \sqrt{\frac{\tau}{2}}$, the grey is the core growth model. In (b) the corresponding trajectories are shown. In the point vortex case, the two vortices translate with uniform speed in the $y$-direction. In the core growth model this speed decays as $1-\exp \left(-\frac{4}{\tau}\right)$. While the speed in the $y$ direction is underestimated by the core growth model, the separation distance between the vorticity extrema is accurately described by $\sqrt{2 \tau}$ as predicted by the core growth model.

Theorem 4. Dynamics of critical points for $\alpha<0$.

- The vortices move away from each other with $|x(\tau)| \leq \frac{\left|x_{0}\right|}{\tau_{0}} \tau$.

- For $\alpha<0, \alpha \neq-1$ the weaker vortex moves to infinity with speed bounded from below by $\frac{1}{4} \ln (-\alpha)$ and the stronger vortex moves towards the center of vorticity.

- For $\alpha=-1$ the vortices asymptotically follow

$$
x(\tau)= \pm \sqrt{\frac{\tau}{2}}, \quad \text { for } \tau \text { large. }
$$

As $|x|>1$ for $\alpha<0, \tau$ must at least be larger than 2 for the above asymptotic solution to hold. From Fig. 7, it seems to be a good approximation to the full model solution shortly thereafter hence the separation distance between the vorticity extrema are well approximated by $\sqrt{2 \tau}$.

The asymptotic behaviour of $x(\tau)$ for large $\tau$ can be found from an Ansatz of the form $x(\tau)=\tau \sum_{n=0}^{\infty} c_{n} \tau^{-n}$. We seek a solution to $\alpha=g(x, \tau)$ for large $\tau$ and $\alpha<0, \alpha \neq-1$. The Ansatz is inserted and coefficients of same order are collected giving for the lowest orders

$$
x(\tau)=\frac{1}{4} \ln (-\alpha) \tau+\frac{2}{\ln (-\alpha)}+\mathcal{O}\left(\tau^{-1}\right) .
$$

Comparison to the vorticity transport equation, Fig. 8, shows an upper bound of the vortex positions in the co-rotating frame is given by the first two terms of this expansion. 
(a)

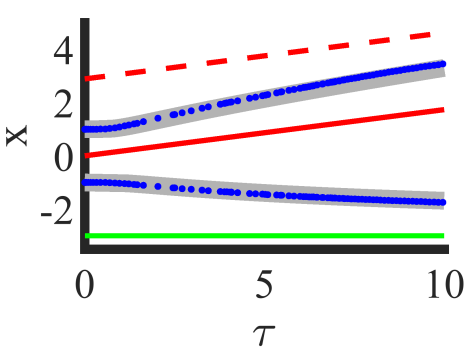

(b)

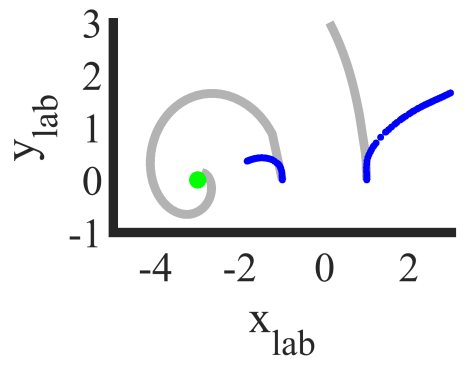

Figure 8: The location of the critical points of vorticity in the co-rotating frame are shown in (a) for $\operatorname{Re}=20, \alpha=-2$. The dashed red line is $x(\tau)=\frac{1}{4} \ln (-\alpha) \tau+\frac{2}{\ln (-\alpha)}$, the full red line is $x(\tau)=\frac{1}{4} \ln (-\alpha) \tau$, blue dots are the solutions to the vorticity transport equation and the green line corresponds to the center of vorticity. In (b) the corresponding trajectories with the green dot being the center of vorticity are shown. Here the model has been integrated for sufficiently long to see one vorticity extremum approach the center of vorticity.

\section{Discussion}

Analytical solutions of the vorticity transport equation are in general very difficult to obtain, but approximate models which allow analytical results may provide useful insight.

Studies of the core growth models are sparse compared to the rich literature on point vortex dynamics where viscous effects are not included. The present, analytical investigation shows that two co-rotating vortices in the core growth model ultimately leads to exactly one extremum of vorticity. This long term solution is in agreement with the important theorem on long time dynamics of the two dimensional vorticity transport equation in Gallay and Wayne [2005].

Two vortices can change into one through a pitchfork bifurcation in the case of equal circulation or through a cusp (perturbed pitchfork bifurcation) in the case of unequal circulations. The topologies of critical points of vorticity are in agreement with numerical results presented here and by e.g. Brandt and Nomura [2007] and with experimental and numerical results by Meunier et al. [2005]. It is a useful feature of the core growth model that it allows for a complete classification of the flow topologies as well as simple, analytical expressions for the bifurcation curves at low Reynolds numbers. Furthermore, simple expressions for the position of all vorticity extrema may be derived. Brøns and Bisgaard [2010] derive a differential equation for the position of critical points of vorticity in viscous flows. In the limit of vanishing viscosity the vorticity extrema are advected with the fluid. For viscous flows a term is added proportional to the viscosity and also depending on high order derivatives of the vorticity.

Jing et al. [2012] numerically compute the merging time in the core growth model with two vortices. Two cases are investigated corresponding to $\alpha=1$ and $\alpha=2$. The used scalings differs from ours, but the ratio $\frac{\tau_{m}(\alpha=1)}{\tau_{m}(\alpha=2)}$ can easily be compared to our results. Excellent agreement is obtained as both approaches give the ratio 0.58. Jing et al. [2012] numerically compute the bifurcation diagram in the core growth model for the two cases. Here, we have found analytical expression of the curves in the bifurcation diagram which are valid for any choice of vortex strengths. 
(a)

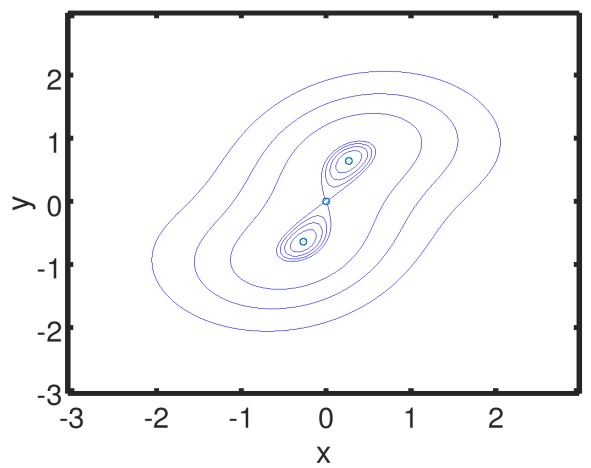

(b)

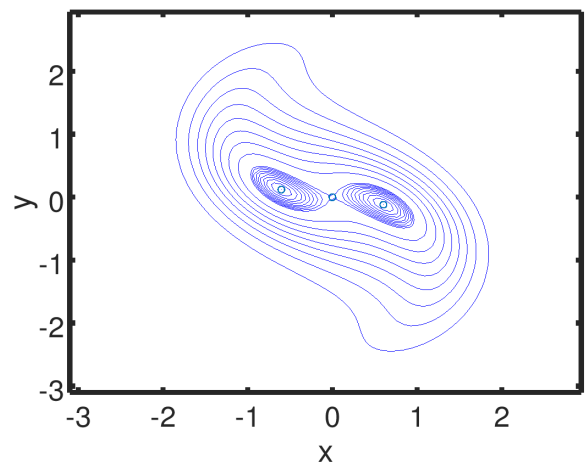

Figure 9: Example of vorticity contours of the vorticity transport equation seen in the laboratory frame for $\alpha=1$. In (a) $R e=50$, in (b) $R e=200$. In the latter case filamentation is more pronounced, a process not included in the core growth model.

Jing et al. [2010] find numerically that the bifurcation time in the core growth model of three collinear vortices depends linearly on Reynolds number, a trend that is also observed for the vorticity transport equation. As shown here, this result is analytically exact for the core growth model of two vortices.

The convective stage of vortex merging is negligible in the viscous situation studied here. Melander et al. [1987], Melander et al. [1988] and Cerretelli and Williamson [2003] relate the convective stage to the filamentation of the vorticity. This leads to asymmetry of the vorticity field which pushes the vortex extrema together. However, one must be careful when inferring from the instantaneous flow field to the transport properties of the fluid i.e. when comparing the Eulerian and Lagrangian flow properties. For vortex patches in inviscid fluid this is discussed by Fuentes [2001]. Fuentes [2005] argue that though filamentation may be a part of the merging process it is not responsible for it. Our present studies of vortex merging at low Reynolds numbers confirm this. Melander et al. [1988], Brandt and Nomura [2006] discuss the importance of the misalignment of the vorticity contours and the streamlines in a corotating frame for a faster merging process.

As seen in Fig. 9 the vorticity field does not exhibit significant filamentation for low Reynolds numbers where the core growth model matches well with the vorticity transport equation.

The interaction of two vortices may occur in the vicinity of other vortices providing a background strain or shear for the interaction. This affects the merging process [Brandt and Nomura, 2007, Trieling et al., 2010, Folz and Nomura, 2014]. Application of the core growth model in this setting may provide useful insights. Another interesting approach is to add mechanisms to the core growth model, making it more realistic, albeit more complex. A way to accomplish this is to use a multi-moment vortex method that allows filamentation of the vorticity contours as done by Nagem et al. [2009] and Uminsky et al. [2012] where the lowest order approximation retrieves the core growth model. 


\subsection{Acknowledgements}

The authors thank Professor Jeppe Dyre, Roskilde University, for useful discussions on the validity of the core growth model.

\section{A Mathematical Proofs}

Proof of Theorem 1.

Proof. For convenience, introduce

$$
\begin{aligned}
& \omega_{1}=\exp \left(-\tau^{-1}\left((x+1)^{2}+y^{2}\right)\right) \\
& \omega_{2}=\exp \left(-\tau^{-1}\left((x-1)^{2}+y^{2}\right)\right) .
\end{aligned}
$$

The partial derivatives of $\omega$ are computed to determine the critical points

$$
\begin{aligned}
& \partial_{x} \omega=-2 \tau^{-1}\left((x+1) \alpha \omega_{1}+(x-1) \omega_{2}\right) \\
& \partial_{y} \omega=-2 \tau^{-1} y\left(\alpha \omega_{1}+\omega_{2}\right) .
\end{aligned}
$$

Solving $\partial_{y} \omega=0$ there are two candidates, but only $y=0$ does not exclude a simultaneous solution of $\partial_{x} \omega=0$. Hence, the critical points are solutions of $\partial_{x} \omega(x, 0)=0$. The second order derivatives are needed to determine the type of critical points.

$$
\begin{aligned}
& \partial_{x y} \omega(x, 0)=0 \\
& \partial_{y y} \omega(x, 0)=-2 \tau^{-1}\left(\alpha \omega_{1}(x, 0)+\omega_{2}(x, 0)\right) .
\end{aligned}
$$

Denoting $x^{*}$ as the critical point value solving $\partial_{x} \omega\left(x^{*}, 0\right)=0$ we get

$$
\partial_{y y} \omega\left(x^{*}, 0\right)=-4 \tau^{-1} \frac{\omega_{2}\left(x^{*}, 0\right)}{x^{*}+1},
$$

which means $\partial_{y y} \omega\left(x^{*}, 0\right)>0$ for $x^{*}<-1$ and $\partial_{y y} \omega\left(x^{*}, 0\right)<0$ for $x^{*}>-1$. Finally,

$$
\partial_{x x} \omega(x, 0)=-2\left(\alpha \tau^{-1} \omega_{1}(x, 0)\left(1-2 \tau^{-1}(x+1)^{2}\right)+\tau^{-1} \omega_{2}(x, 0)\left(1-2 \tau^{-1}(x-1)^{2}\right)\right)
$$

For $\alpha>0, \partial_{x} \omega(x, 0)>0$ for $x \leq-1$ and $\partial_{x} \omega(x, 0)<0$ for $x \geq 1$. Hence, all critical points are in the interval $(-1,1)$ and at least one exists for all values of $\tau$ as $\partial_{x} \omega(x, 0)$ crosses the $x$ - axis from positive to negative values. In the typical case of this happening transversally, $\partial_{x x} \omega\left(x^{*}, 0\right)>0$ at the (first) critical point. From Eq. (40) it is clear that $\partial_{y y} \omega\left(x^{*}, 0\right)<0$ for $\alpha>0$. This means that in the case of $\partial_{x} \omega(x, 0)$ crossing the $x$-axis transversally, there will be an odd number of critical points, the first being a extremum and then of alternating type. Similar argumentation applies to $\alpha<0$.

Proof of Theorem 2. 
Proof. $\partial_{x} g(x, \tau)$ has at most two zeros, located at $|x|<1$ due to Eq. (22). Together with the limits (42), the first and last part of the Theorem is proved as $g$ is positive only for $x \in(-1,1)$. Since

$$
\begin{aligned}
\lim _{x \rightarrow \infty} g(x, \tau) & =-\infty \\
\lim _{x \rightarrow-\infty} g(x, \tau) & =0 \\
\lim _{x \rightarrow-1^{ \pm}} g(x, \tau) & = \pm \infty,
\end{aligned}
$$

the range of $g$ on the subdomain $(-1,1)$ is $(0, \infty)$ so there must exist a critical point for any nonzero $\alpha$. Similarly there must exist two critical points for negative $\alpha$. Since $g$ is monotone for $x \in(-\infty,-1)$ and $x \in(1, \infty)$ there are exactly two critical points of the vorticity for negative $\alpha$.

The maximal merging time for any positive $\alpha$ follows from Eq. (23). As the type of the critical points of vorticity is known by Thm. 1, the bifurcation types at merging is a pitchfork for $\alpha=1$ and a perturbed pitchfork for $\alpha \neq 1$.

\section{Proof of Theorem 3.}

Proof. The initial locations of the two extrema are in two distinct trapping regions by Eq. (23). The initial location of the saddle then determines which extremum it will bifurcate with. Since $g$ is increasing from $x_{-}$to $x_{+}$and $g(0, \tau)=1$ then for $\alpha>1, \alpha=g(x, \tau)$ is fulfilled for some $x>0$. Thus, in this case the saddle will merge with the extremum located at $x>0$. Similar arguments apply for $0<\alpha<1$.

Proof of Theorem 4

Proof. Since $\frac{d x}{d \tau}$ is positive for $x>1, \tau>0$ and negative for $x>1, \tau>0$ the two vortex extrema moves away from each other for increasing time for $\alpha<0$.

Considering $x(\tau)>1$ then by Eq. (31)

$$
\frac{d x}{d \tau}<\frac{x}{\tau}
$$

which implies

$$
x(\tau) \leq \frac{x_{0}}{\tau_{0}} \tau .
$$

Similar considerations can be done for the critical point located at $x<-1$ proving the first part of the Theorem.

Since $\frac{x+1}{x-1}$ approaches 1 for large $x$, an estimate can be constructed from Eq. (34) where $\ln \left(\frac{x+1}{x-1}\right)$ is left out. Solving for $x$ gives

$$
x(\tau) \approx \frac{\ln (-\alpha)}{4} \tau, \quad \text { for large } \tau, \alpha<0, \alpha \neq-1
$$

For $\alpha<-1, \ln (-\alpha)$ and $\ln \left(\frac{x+1}{x-1}\right)$ are both positive which means the bound

$$
x(\tau) \geq \frac{\ln (-\alpha)}{4} \tau
$$


holds for any $\tau \geq \frac{4}{\ln (-\alpha)}$. Hence, the positive solution occuring for $x>1$ is bounded below by a linear growth.

The solution for $x<-1$ can be investigated using that $\tau$ must be non negative in Eq. (34), meaning the denominator must be negative giving the criterion

$$
x_{c v}<x<-1 \text {, }
$$

with $x_{c v}$ given by Eq. (27). Since $\frac{d x}{d \tau}<0$ for $x<-1$ by Eq. (31), the stronger vortex will move towards the center of vorticity, $x_{c v}$. The case $0>\alpha>-1$ is covered by the symmetry given in Eq. (19), establishing the Theorem.

Reorganizing Eq. (34b) and using the power series expansion of ln facilitates investigation of the dynamics for large $\tau$ and $\alpha=-1$

$$
\ln \left(\frac{x+1}{x-1}\right)=\ln \left(1+\frac{2}{x-1}\right)=\frac{2}{x-1}-\frac{1}{2}\left(\frac{2}{x-1}\right)^{2}+\ldots
$$

For large $|x|$ only the lowest order of the expansion is used

$$
\tau=\frac{4 x}{\ln (-\alpha)+\frac{2}{x-1}} .
$$

Then, for large $|x|$ and $\alpha=-1$

$$
x(\tau)= \pm \sqrt{\frac{\tau}{2}}, \quad \text { for } \tau \text { large. }
$$

\section{References}

H. Aref. Motion of three vortices. Physics of Fluids, 22(3):393-400, 1979. doi: $10.1063 / 1.862605$.

A. Balci, M. Andersen, M. C. Thompson, and M. Brøns. Codimension three bifurcation of streamline patterns close to a no-slip wall: A topological description of boundary layer eruption. Physics of Fluids, 27(5):053603, 2015. doi: 10.1063/1.4921527.

J. T. Beale and A. Majda. Rates of convergence for viscous splitting of the Navier-Stokes equations. Mathematics of Computation, 37:243 - 259, 1981.

L. K. Brandt and K. K. Nomura. The physics of vortex merger: Further insight. Physics of Fluids, 18(5):051701, 2006. doi: 10.1063/1.2201474.

L. K. Brandt and K. K. Nomura. The physics of vortex merger and the effects of ambient stable stratification. Journal of Fluid Mechanics, 592:413-446, 2007.

L. K. Brandt and K. K. Nomura. Characterization of the interactions of two unequal corotating vortices. Journal of Fluid Mechanics, 646:233-253, 2010.

M. Brøns. Streamline topology: Patterns in fluid flows and their bifurcations. volume 41 of Advances in Applied Mechanics, pages 1 - 42. Elsevier, 2007. doi: https://doi.org/10.1016/S0065-2156(07)41001-8. 
M. Brøns and Anders V. Bisgaard. Topology of vortex creation in the cylinder wake. Theoretical and Computational Fluid Dynamics, 24(1):299-303, Mar 2010. ISSN 1432-2250. doi: 10.1007/s00162-009-0110-0.

M. Brøns, B. Jakobsen, K. Niss, A. Bisgaard, and L. K. Voigt. Streamline topology in the near wake of a circular cylinder at moderate Reynolds numbers. Journal of Fluid Mechanics, 584:23-43, 2007. ISSN 0022-1120. doi: 10.1017/S0022112007006234.

C. Cerretelli and H.K. Williamson. The physical mechanism for vortex merging. Journal of Fluid Mechanics, 475:4177, 2003. doi: 10.1017/S0022112002002847.

G. S. Deem and N. J. Zabusky. Vortex waves: Stationary " $v$ states," interactions, recurrence, and breaking. Physical Review Letters, 40:859-862, Mar 1978. doi: 10.1103/PhysRevLett.40.859.

D. G. Dritschel. The stability and energetics of corotating uniform vortices. Journal of Fluid Mechanics, 157:95134, 1985. doi: 10.1017/S0022112085002324.

W. E and J. G. Liu. Vorticity boundary conditions and related issues for finite difference schemes. Journal of Computational Physics, 124:368-382, 1996a.

W. E and J.G. Liu. Finite difference schemes for incompressible flows in vorticity formulations. ESIAM: Proceedings, 1:181-195, 1996b.

J. W. Eaton, D. Bateman, S. Hauberg, and R. Wehbring. GNU Octave version 4.2.0 manual: a high-level interactive language for numerical computations. CreateSpace Independent Publishing Platform, 2016.

P. J. R. Folz and K. K. Nomura. Interaction of two equal co-rotating viscous vortices in the presence of background shear. Fluid Dynamics Research, 46:12, 2014.

P. J. R Folz and K. K. Nomura. A quantitative assessment of viscous asymmetric vortex pair interactions. Journal of Fluid Mechanics, 829:1-30, 2017.

O. V. Fuentes. Chaotic advection by two interacting finite-area vortices. Physics of Fluids, 13(4):901-912, 2001. doi: 10.1063/1.1352626.

O. V. Fuentes. Vortex filamentation: Its onset and its role on axisymmetrization and merger. Dynamics of Atmospheres and Oceans, 40:23-42, 2005.

T. Gallay. Interaction of vortices in weakly viscous planar flows. Archive for Rational Mechanics and Analysis, 200(2):445-490, 2011.

T. Gallay and C. E. Wayne. Global stability of vortex solutions of the two-dimensional Navier-Stokes equation. Communications in Mathematical Physics, 255(1):97-129, 2005.

G. Haller. An objective definition of a vortex. Journal of Fluid Mechanics, 525:126, 2005. doi: $10.1017 /$ S0022112004002526.

G. Haller, A. Hadjighasem, M. Farazmand, and F. Huhn. Defining coherent vortices objectively from the vorticity. Journal of Fluid Mechanics, 795:136-173, 2016. 
J. S. Hansen. GNU Octave - Beginner's Guide. Packt Publiching, London, 2011.

M. Heil, J. Rosso, A. L. Hazel, and M. Brøns. Topological fluid mechanics of the formation of the Kármán-vortex street. Journal of Fluid Mechanics, 812:199-221, 2017.

L. Jacquin, D. Fabre, D. Sipp, V. Theofilis, and H. Vollmers. Instability and unsteadiness of aircraft wake vortices. Aerospace Science and Technology, 7:577-593, 2003.

J. Jeong and F. Hussain. On the identification of a vortex. Journal of Fluid Mechanics, 285: 6994, 1995. doi: 10.1017/S0022112095000462.

F. Jing, E. Kanso, and P. K. Newton. Viscous evolution of point vortex equilibria: The collinear state. Physics of Fluids, 22:123102, 2010.

F. Jing, E. Kanso, and P. K. Newton. Insights into symmetric and asymmetric vortex mergers using the core growth model. Physics of Fluids, 24:073101, 2012.

C. Josserand and M. Rossi. The merging of two co-rotating vortices: a numerical study. European Journal of Mechanics - B/Fluids, 26(6):779 - 794, 2007. ISSN 0997-7546. doi: https://doi.org/10.1016/j.euromechflu.2007.02.005.

T. Kida and T. Nakajima. Core spreading vortex methods in two-dimensional viscous flows. Computer Methods in Applied Mechanics and Engineering, 160(3):273 - 298, 1998. ISSN 0045-7825. doi: https://doi.org/10.1016/S0045-7825(97)00298-3.

S. Kim and S. Sohn. Interactions of three viscous point vortices. Journal of Physics A: Mathematical and Theoretical, 45(45):455501, 2012.

T Leweke, S. Le Dizes, and Charles H.K. Williamson. Dynamics and instabilities of vortex pairs. Annual Review of Fluid Mechanics, 48(1):507-541, 2016. doi: 10.1146/annurev-fluid122414-034558.

C. Marchioro. On the inviscid limit for a fluid with a concentrated vorticity. Commun Math Phys, 193:53-65, 1998.

M. V. Melander, J. C. Mcwilliams, and N. J. Zabusky. Axisymmetrization and vorticitygradient intensification of an isolated two-dimensional vortex through filamentation. Journal of Fluid Mechanics, 178:137159, 1987. doi: 10.1017/S0022112087001150.

M. V. Melander, N. J. Zabusky, and J. C. Mcwilliams. Symmetric vortex merger in two dimensions: causes and conditions. Journal of Fluid Mechanics, 195:303340, 1988. doi: $10.1017 /$ S0022112088002435.

V. V. Meleshko and H. Aref. A bibliography of vortex dynamics 1858-1956. Advances in Applied Mechanics, 41, 2007. ISSN 0065-2156.

P. Meunier, U. Ehrenstein, T. Leweke, and M. Rossi. A merging criterion for two-dimensional co-rotating vortices. Physics of Fluids, 2002:2757-2766, 2002.

P. Meunier, S. Le Cizes, and T. Leweke. Physics of vortex merging. C. R. Physique, 2005: 431-450, 2005. 
R. Nagem, G. Sandri, D. Uminsky, and C. E. Wayne. Generalized Helmholtz - Kirchhoff model for two-dimensional distributed vortex motion. SIAM Journal on Applied Dynamical Systems, 8(1):160-179, 2009. doi: 10.1137/080715056.

P. K. Newton. The N-vortex problem, analytical techniques, volume vol. 145 of Applied mathematical sciences vol. 145. Springer, New York, 2001. ISBN 0387952268.

P. G. Saffman. Vortex dynamics. Cambridge University Press, 1992.

J. L. Synge. On the motion of three vortices. Can. J. Math, 1:257-270, 1949.

M. Tobak and D. J. Peake. Topology of three-dimensional separated flows. Annual Review of Fluid Mechanics, 14(1):61-85, 1982. doi: 10.1146/annurev.fl.14.010182.000425.

R. R. Trieling, O. U. V. Fuentes, and G. J. F. van Heijst. Interaction of two unequal corotating vortices. Physics of Fluids, 17(8):087103, 2005. doi: 10.1063/1.1993887.

R. R. Trieling, C. E. C. Dam, and G. J. F. van Heijst. Dynamics of two identical vortices in linear shear. Physics of Fluids, 22(11):117104, 2010. doi: 10.1063/1.3489358.

D. Uminsky, C. E. Wayne, and A. Barbaro. A multi-moment vortex method for $2 \mathrm{~d}$ viscous fluids. Journal of Computational Physics, 231(4):1705 - 1727, 2012. ISSN 0021-9991. doi: https://doi.org/10.1016/j.jcp.2011.11.001. 